\title{
Exact embeddings of JT gravity in strings and M-theory
}

\author{
Yue-Zhou Li ${ }^{1, \mathrm{a}}$, Shou-Long $\mathbf{L i}^{2, \mathrm{~b}}$, H. Lï ${ }^{1, \mathrm{c}}$ \\ ${ }^{1}$ Department of Physics, Tianjin University, Tianjin 300350, China \\ ${ }^{2}$ School of Physics, Beijing Institute of Technology, Beijing 100081, China
}

Received: 3 August 2018 / Accepted: 20 September 2018 / Published online: 28 September 2018

(C) The Author(s) 2018

\begin{abstract}
We show that two-dimensional JT gravity, the holographic dual of the IR fixed point of the SYK model, can be obtained from the consistent Kaluza-Klein reduction of a class of EMD theories in general $D$ dimensions. For $D=4,5$, the EMD theories can be themselves embedded in supergravities. These exact embeddings provide the holographic duals in the framework of strings and M-theory. We find that a class of JT gravity solutions can be lifted to become time-dependent charged extremal black holes. They can be further lifted, for example, to describe the D1/D5branes where the worldsheet is the Milne universe, rather than the typical Minkowski spacetime.
\end{abstract}

\section{Contents}

1 Introduction $\ldots \ldots \ldots \ldots \ldots \ldots$

2 An EMD embedding of JT gravity . . . . . . . . 2

2.1 A class of EMD theories . . . . . . . . . . 2

2.2 Conformal transformation . . . . . . . . 3

2.3 JT gravity from Kaluza-Klein reduction ... . 3

3 Oxidations and time-dependent black holes . . . . . 5

3.1 Oxidation of the solutions . . . . . . . . . 5

3.2 Time-dependent extremal black holes ... . . 5 5

4 Embeddings in strings and M-theory ... . . . . 6

4.1 The $D=5$ theory . . . . . . . . . . . 6

4.2 The $D=4$ theory . . . . . . . . . . . 6

$4.3 D=4,5$ theories with $g_{1} \neq 0 \ldots \ldots$

5 Conclusions .............. 7

A From JT gravity to SYK . . . . . . . . . . . . . . 8

References.................. . . 9

\footnotetext{
a e-mail: liyuezhou@tju.edu.cn

b e-mail: sllee_phys@bit.edu.cn

ce-mail: mrhonglu@gmail.com
}

\section{Introduction}

The AdS/CFT correspondence [1,2] serves as a bridge that connects some conformal field theory $\left(\mathrm{CFT}_{d}\right)$ in $d$ dimensions and gravity in the anti-de Sitter $\left(\operatorname{AdS}_{D}\right)$ background in $D=d+1$ dimensions. This holographic duality was best studied between the $\mathcal{N}=4 D=4$ superconformal field theory and type IIB string in the $\mathrm{AdS}_{5} \times S^{5}$ background. The duality is however expected to be applicable for wider classes of theories, possibly even beyond conformal field theories. The duality remains largely conjectural, and the simplest examples to prove this duality may be associated with the integrable models that can be solved completely. However, such models with conformal symmetries are hard to come by.

Recently, Sachdev-Ye-Kitaev (SYK) model [3-5], which describes random all-to-all interactions between $N$ Majorana fermions in $0+1$ dimension, has drawn a large amount of attentions due to its integrability in the large $N$ limit. The SYK model exhibits approximate conformal symmetry in the infrared (IR) limit, suggesting that the SYK model may be a $\mathrm{CFT}_{1}$ at low energy. It was shown to be maximally chaotic [4-6] in the sense that its out-of-time order correlators exhibit Lyapunov exponents and butterfly effects [7] and they saturate [8] the chaos bound established by black holes [7]. Therefore, the IR limit of the SYK model may have an $\mathrm{AdS}_{2}$ bulk gravity dual.

However, the naive $\mathrm{CFT}_{1}$ interpretation of the SYK model is not appropriate. For the standard $\mathrm{CFT}_{1}$ interpretation, the SYK model should encode the full Virasoro algebra in the IR limit and exhibit the time reparametrization invariance. Instead the time reparametrization symmetry is spontaneously broken into its $S L(2, \mathbb{R})$ subgroup, giving rise to Goldstone zero modes [5]. The effective theory is described by a Scwharzian action. This fact demonstrates that the model deviates from the standard $\mathrm{CFT}_{1}$ and becomes the $0+1$ dimensional "nearly conformal field theory" (NCFT) [5]. Many properties of $\mathrm{SYK}_{1}$ and $\mathrm{NCFT}_{1}$ have been explored, in 
e.g. [6,9-22]. The supersymmetric generalizations [23-27] and higher dimensional generalizations [28-30] were also constructed.

The $\mathrm{NCFT}_{1}$ property is related to the fact that the bulk theory can not be Einstein gravity in $D=2$ dimensions. Specifically, the Einstein-Hilbert action

$S=\frac{1}{16 \pi} \int d^{2} x \sqrt{-g} R$.

is simply a topological constant and thus gives no dynamics. Thus nontrivial $D=2$ gravity must non-minimally couple to a matter field. The simplest example is perhaps the JackiwTeitelboim (JT) gravity [31,32]

$S=\frac{1}{16 \pi} \int d^{2} x \sqrt{-g} \Phi\left(R-2 \Lambda_{0}\right)$.

When $\Lambda_{0}$ is negative, the JT model admits the $\mathrm{AdS}_{2}$ vacuum; however, the full $\mathrm{AdS}_{2}$ symmetry is broken by the nontrivial dilaton $\Phi$ [33]. JT gravity may thus provide a gravity dual of the IR limit of the SYK model [34] (see Appendix A for details), and the $S Y K / A_{d} S_{2}$ duality can thus be addressed in the context of JT gravity [15,33-40].

In fact, one may consider more complicated dilaton gravities in two dimensions, which were extensively studied in the last century for addressing basic problems of quantum gravity (see, e.g. [41] for a review.) Two-dimensional gravities received new attention in the light of holography. Almheiri and Polchinski (AP) recently introduced a general family of dilaton-gravity models [42]

$S=\frac{1}{16 \pi} \int d^{2} x \sqrt{-g}\left(\tilde{\Phi}^{2} R+\lambda(\partial \tilde{\Phi})^{2}-U(\tilde{\Phi})\right)$.

For appropriate potential $U$, the theory admits $\mathrm{AdS}_{2}$ vacuum with constant $\tilde{\Phi}^{2}=\Phi_{0}$. One can now perform a perturbation,

$\tilde{\Phi}^{2}=\Phi_{0}+\Phi, \quad \Phi \ll \Phi_{0}$,

the effective action for the linear perturbation is then precisely the JT gravity [43].

The AP class of models can be obtained from higher dimensional theories such as strings and M-theory via Kaluza-Klein reductions. This provides an understanding of SYK models from the higher dimensional point of view. Indeed, many higher dimensional extremal black holes has near horizon geometries as an $\mathrm{AdS}_{2} \times \mathcal{M}$ [44-47,49-52], and the near horizon region can be effectively described by $D=2$ dilaton gravities (1.3) in many situations [43-48].

However, the above embeddings of JT gravity is at the linear perturbation level. From the higher-dimensional point of view, $\mathrm{AdS}_{2}$ spacetimes typically arise from the near-horizon geometry of some extremal black holes. Thus JT gravity as a linear perturbation of the AP class describes the leadingorder approximation away from the extremality. Since JT gravity itself only captures the IR behavior of SYK models, these embeddings have to deal with the subtleties associated with these two competing approximations. In this paper we seek exact embeddings of JT gravity in higher dimensions so that the leading-order approximation away from the extremality has a broader range of validity. The simplest example is perhaps Einstein gravity with a cosmological constant in three dimensions, with the reduction ansatz being $d s_{3}^{2}=d s_{2}^{2}+\Phi^{2} d z^{2}[14,53-56]$. Since $\mathrm{AdS}_{3}$ emerges naturally in strings and $\mathrm{M}$-theory, the reduction ansatz provides a direct link between SYK and string theories. Ref. [53] also obtained JT gravity coupled to a Maxwell field from the STU supergravity model in four dimensions [57] by the KaluzaKlein reduction on $S^{2}$.

In this paper, we present an alternative exact embedding of JT gravity in higher dimensions. We construct a class of Einstein-Maxwell-Dilaton (EMD) theories in general $D$ dimensions with appropriate dilaton couplings and scalar potential. We demonstrate that JT gravity can be obtained from the EMD theories via consistent Kaluza-Klein reductions. It turns out that for $D=4$ and $D=5$, the EMD theories without the scalar potential can be embedded in supergravities, which themselves can be obtained from the Kaluza-Klein reduction of strings and M-theory.

The paper is organized as follows. In Sect. 2, we present a class of EMD theories in $D$ dimensions and express them in the $f(R)$-frame where the manifest kinetic term of the dilaton vanishes. We then perform consistent Kaluza-Klein reductions and show that JT gravity can indeed emerge. In Sect. 3, we consider solutions in JT gravity and oxidize them to become solutions in the EMD theories in higher dimensions. We find that a class of JT gravity solutions are related to the previously-known time-dependent extremal black holes. In Sect. 4, we consider the EMD theories in four and five dimensions and show they are consistent truncations of the bosonic sector of supergravities and/or gauged supergravities. This allows to embed the solutions in strings and Mtheory. We conclude the paper in Sect. 5. In the appendix, we give some detail review of how JT gravity can give rise to the Schwarzian action.

\section{An EMD embedding of JT gravity}

\subsection{A class of EMD theories}

We begin with a class of EMD theories considered in [58]. The theories consist of the metric, a scalar $\phi$, and two $U(1)$ gauge fields $A$ and $\mathcal{A}$. The Lagrangian in the Einstein frame is given by 
$\mathcal{L}=\sqrt{-g}\left(R-\frac{1}{2}(\partial \phi)^{2}-V(\phi)-\frac{1}{4} e^{a \phi} F^{2}-\frac{1}{4} e^{b \phi} \mathcal{F}^{2}\right)$,

where $F=d A, \mathcal{F}=d \mathcal{A}$ and the dilatonic coupling constants satisfy

$a b=-\frac{2(D-3)}{D-2}$.

The scalar potential $V$ is inspired by those in gauge supergravities, given in terms of a super-potential $W$ [58]

$$
\begin{aligned}
V & =\left(\frac{d W}{d \phi}\right)^{2}-\frac{D-1}{2(D-2)} W^{2}, \\
W & =\frac{\sqrt{2}(D-2)^{2} a}{(D-2) a^{2}+2(D-3)} g\left(b e^{-\frac{1}{2} a \phi}-a e^{-\frac{1}{2} b \phi}\right) .
\end{aligned}
$$

For reasons that will become apparent, in this paper, we are particularly interested in the case with

$a=\sqrt{\frac{2(D-3)^{2}}{(D-1)(D-2)}}, \quad b=-\sqrt{\frac{2(D-1)}{D-2}}$.

The potential is thus

$$
\begin{aligned}
V & =-(D-1) g^{2}\left(e^{-\frac{2(D-3) \phi}{\sqrt{2(D-1)(D-2)}}}+(D-3) e^{\frac{2 \phi}{\sqrt{2(D-1)(D-2)}}}\right) \\
& =-(D-1) g^{2}\left(e^{-a \phi}+(D-3) e^{-\frac{1}{2}(a+b) \phi}\right) .
\end{aligned}
$$

Note that if we set $A=0$ and also turn off the scalar potential, the remainder of the theory is simply the Kaluza-Klein theory with $\mathcal{A}$ being the Kaluza-Klein vector. The EMD theories were inspired by gauged supergravities. In $D=4$ and 5, the Lagrangians are the consistent truncations of the bosonic sector of the respective gauged STU models. Their embeddings in M-theory and type IIB strings via Kaluza-Klein sphere reductions were given in [59].

We now make a constant shift of $\phi$, and redefine

$$
\begin{aligned}
& \phi=\tilde{\phi}+c, \quad A_{\mu}=\tilde{A}_{\mu} e^{-\frac{1}{2} a c}, \quad \mathcal{A}_{\mu}=\tilde{\mathcal{A}}_{\mu} e^{-\frac{1}{2} b c}, \\
& g^{2} e^{-a c}=g_{2}^{2}, \quad e^{-\frac{1}{2}(a+b) c} g^{2}=g_{1}^{2} .
\end{aligned}
$$

Dropping the tilde, the Lagrangian takes the same form as (2.1), but with $V$ now given by

$$
V=-(D-1)\left(g_{2}^{2} e^{-a \phi}+(D-3) g_{1}^{2} e^{-\frac{1}{2}(a+b) \phi}\right) .
$$

This allows us to set the parameter $g_{1}$ and $g_{2}$ to zero independently.

\subsection{Conformal transformation}

We now make a conformal transformation [60]

$$
g_{\mu \nu}=e^{-\sqrt{\frac{2}{(D-1)(D-2)}} \phi} \tilde{g}_{\mu \nu},
$$

the Lagrangian, after dropping the tildes, becomes

$$
\begin{aligned}
\mathcal{L}= & \sqrt{-g}\left(\Phi\left(R+(D-1)(D-3) g_{1}^{2}\right)-\frac{1}{4} \Phi^{-1} F^{2}\right. \\
& \left.+\Phi^{3}\left((D-1) g_{2}^{2}-\frac{1}{4} \mathcal{F}^{2}\right)\right) .
\end{aligned}
$$

where

$\Phi=e^{-\sqrt{\frac{D-2}{2(D-1)}} \phi}$.

The conformal transformation (2.8) is such that the dilaton's kinetic term is absent and the equation of motion for $\Phi$ becomes algebraic. This can be generally done in supegravities or gauged supergravities and such a conformally transformed theory was referred as the $f(R)$-version of supergravity in [60]. In this paper we shall also refer (2.9) as gravity in the $f(R)$-frame.

In order to make contact with JT gravity through dimensional reduction, we take $\mathcal{A}=0$ and $g_{2}=0$, the resulting EMD theory in the $f(R)$-frame is

$\mathcal{L}=\sqrt{-g}\left(\Phi\left(R+(D-1)(D-3) g_{1}^{2}\right)-\frac{1}{4} \Phi^{-1} F^{2}\right)$.

In four and five dimensions, the theories can be obtained from taking appropriate limit of the bosonic sector of the STU gauged supergravity models. When $g_{1}=0$, they can be truncated consistently from supergravities and hence can be embedded in string and M-theory.

\subsection{JT gravity from Kaluza-Klein reduction}

In this subsection, we show that JT gravity can be obtained from this EMD theory (2.11) by the consistent KaluzaKlein reduction. The internal space is taken to be $(D-2)$ dimensional Einstein-space $d \Omega_{D-2, k}^{2}$ with $R_{i j}=(D-$ 3) $k g_{i j}$, where $k=-1,0,1$. The corresponding Ricci scalar is given by

$R_{k}=(D-2)(D-3) k$

In order for the reduction to be consistent, we take all the singlet of the isometry group of the internal space. The reduction ansatz is thus given by

$d s_{D}^{2}=d s_{2}^{2}+\varphi(x)^{2} d \Omega_{D-2, k}^{2}, \quad A_{\mu}=A_{\mu}(x), \quad \Phi=\Phi(x)$,

where $\mu=0,1$ and $x^{\mu}$ are respectively the indices and coordinates of the metric $d s_{2}^{2}$. The Kaluza-Klein reduction in the Einstein frame down to dimensions higher than or equal to three was obtained in [61]. We find that the reduced Lagrangian from (2.11) in two dimensions is 


$$
\begin{aligned}
\mathcal{L}_{2}= & \sqrt{-g} \Phi \varphi^{D-2}\left(R+\varphi^{-2} R_{k}\right. \\
& -(D-2)(D-3) \varphi^{-2}(\partial \varphi)^{2}-2(D-2) \varphi^{-1} \square \varphi \\
& \left.+(D-1)(D-3) g_{1}^{2}-\frac{1}{4} \Phi^{-2} F^{2}\right) .
\end{aligned}
$$

The equation of motion associated with $A$ is

$$
\nabla_{\mu}\left(\Phi^{-1} \varphi^{D-2} F^{\mu \nu}\right)=0,
$$

which can be solved by

$$
F=\lambda \Phi \varphi^{-(D-2)} \epsilon_{(2)},
$$

where $\lambda$ is an integration constant associated with the electric charge and $\epsilon_{(2)}$ is the volume 2-form of $d s_{2}^{2}$. The variations associated with $\Phi$ and $\varphi$ yield

$$
\begin{aligned}
0= & R+\varphi^{-2} R_{k}-(D-2)(D-3) \varphi^{-2}(\partial \varphi)^{2} \\
& -2(D-2) \varphi^{-1} \square \varphi \\
& +(D-1)(D-3) g_{1}^{2}+\frac{1}{4} \Phi^{-2} F^{2}, \\
0= & 4 R_{k} \Phi^{2}+(D-2)\left(\varphi^{2} F^{2}\right. \\
& +4 \Phi\left(-\Phi \varphi \square \varphi+\varphi^{2} \square \Phi\right. \\
& \left.\left.+(D-3)\left(\varphi \nabla_{\mu} \varphi \nabla^{\mu} \Phi-\Phi(\partial \varphi)^{2}\right)\right)\right) .
\end{aligned}
$$

Finally, the equation of motion associated with the variation of $g_{\mu \nu}$, when (2.17) and (2.18) are applied, is given by

$$
\begin{aligned}
& \Phi^{2} \varphi R_{\mu \nu}+\Phi\left(\varphi \square \Phi+(D-2) \nabla_{\rho} \varphi \nabla^{\rho} \Phi\right) g_{\mu \nu} \\
& \quad+\frac{1}{4} \varphi\left(F^{2} g_{\mu \nu}-2 F_{\mu \rho} F_{\nu}^{\rho}\right) \\
& \quad-\Phi\left(\varphi \nabla_{\mu} \nabla_{\nu} \Phi+(D-2) \Phi \nabla_{\mu} \nabla_{\nu} \varphi\right)=0 .
\end{aligned}
$$

Taking the trace yields

$$
\Phi \varphi R+\varphi \square \Phi+2(D-2) \nabla_{\rho} \Phi \nabla^{\rho} \varphi-(D-2) \Phi \square \varphi=0 .
$$

This equation, together with the two scalar equations (2.17) and (2.18), imply that we can take $\varphi=\varphi_{0}$ to be constant, provided that the charge parameter $\lambda$ is

$\lambda^{2}=(D-1)(D-3)\left(k+g_{1}^{2} \varphi_{0}^{2}\right) \varphi_{0}^{2(D-3)}$.

The remainder equations can be summarized as

$$
R-2 \Lambda_{0}=0, \quad R_{\mu \nu} \Phi+g_{\mu \nu} \square \Phi-\nabla_{\mu} \nabla_{\nu} \Phi=0 .
$$

where

$$
\Lambda_{0}=-\frac{1}{4}(D-3)\left(k(D-3) \varphi_{0}^{-2}+(D-1) g_{1}^{2}\right) .
$$

It is now straightforward to see that the Eq. (2.22) can be derived from the action of JT gravity (1.2). In Appendix A, we review how the JT action, together with the bounary terms of the Gibbons-Hawking type and the holographic counterterm, give rise to the Schwarzian action in the appropriate $\mathrm{AdS}_{2}$ background. (A more general argument for how the Schwarzian action arises from AP models was presented in [53].)

To conclude, JT gravity with (2.23) can be obtained from the Kaluza-Klein reduction of the $D$-dimensional EMD theory (2.11) and the consistent reduction ansatz is

$$
\begin{aligned}
d s_{D}^{2} & =d s_{2}^{2}+\varphi_{0}^{2} d \Omega_{D-2, k}^{2}, \\
F & =\sqrt{(D-1)(D-3)\left(k+g_{1}^{2} \varphi_{0}^{2}\right)} \varphi_{0}^{-1} \Phi \epsilon_{(2)} .
\end{aligned}
$$

In the case of $D=3$, nontrivial results require the absorbing of the $(D-3)$ factor into $g_{1}^{2}$. It is instructive simply to introduce $\tilde{g}_{1}^{2}=(D-3) g_{1}^{2}$ and declare that $\tilde{g}_{1}^{2}$ is non-vanishing in $D=3$. To be specific, we see that the $D=3$ theory in the Einstein frame is

$\mathcal{L}_{3}=\sqrt{-g}\left(R-\frac{1}{2}(\partial \varphi)^{2}+2 \tilde{g}_{1}^{2} e^{\varphi}-\frac{1}{4} F^{2}\right)$.

In the $f(R)$-frame, it becomes

$\mathcal{L}_{3}=\sqrt{-g}\left[\Phi\left(R+2 \tilde{g}_{1}^{2}\right)-\frac{1}{4} \Phi^{-1} F^{2}\right]$.

The reduction ansatz from $D=3$ to $D=2$ is

$d s_{3}^{2}=d s_{2}^{2}+\varphi_{0}^{2} d z^{2}, \quad F=\sqrt{2} \tilde{g}_{1} \Phi \epsilon_{(2)}$.

The resulting two-dimensional theory is then the JT theory (1.2) with $\Lambda_{0}=-\frac{1}{2} \tilde{g}_{1}^{2}$. Note that equations of motion of the resulting JT gravity are independent of the constant parameter $\varphi_{0}$.

The $D=3$ case perfectly illustrated the difference between our embedding of JT gravity in higher dimensions and those discussed previously in literature. In [14], (also see [53-56],) the higher-dimensional theory is pure AdS gravity and the scalar in JT gravity arises as the radius of the compactifying circle $z$. The running of the JT scalar is driven by this breathing mode of the internal space. On the other hand, in our embedding, the internal radius is fixed consistently by the equations of motion to be a constant. The JT scalar is a direct descendant of the dilaton in higher dimensions. Both embeddings are possible due to the fact that JT gravity is not conformal in that it has a running dilaton, which may arise directly from the higher-dimensional theory or from the modulus parameter of the compactifying space in a theory that has no scalar. 


\section{Oxidations and time-dependent black holes}

In the previous section, we demonstrate that JT gravity in two dimensions can be obtained from the consistent KaluzaKlein reduction of the EMD theory (2.11) on an internal Einstein space. This allows us to oxidize all the two-dimensional solutions to higher dimensions. In particular, we find that some special two-dimensional solutions become the decoupling limit of time-dependent extremal black holes.

\subsection{Oxidation of the solutions}

The two-dimensional metric is Einstein with a negative cosmological constant. We can thus take the metric to be

$d s^{2}=-f(r) d t^{2}+\frac{1}{f(r)} d r^{2}$.

Then Eq. (2.22) of JT gravity admit the locally $\mathrm{AdS}_{2}$ solution

$$
f=-\Lambda_{0} r^{2}, \quad \Phi=\frac{\alpha}{r}+\left(\beta+\gamma t-\Lambda_{0}^{2} \alpha t^{2}\right) r,
$$

where $\alpha, \beta, \gamma$ are integral constants. The theory also admits the black hole solution

$$
\begin{aligned}
d s^{2}= & \frac{1}{z^{2}}\left(\frac{d z^{2}}{f}-f d t^{2}\right), \quad f=1-\frac{z^{2}}{z_{0}^{2}} \\
\Phi= & -\frac{2 c_{2} z_{0}^{2}}{z}(1-\sqrt{f})+\frac{\sqrt{f}}{z}\left(z_{0}\left(c_{1} \sinh \left(\frac{t}{z_{0}}\right)-2 c_{2} z_{0}\right)\right. \\
& \left.+\left(2 c_{2} z_{0}^{2}+c_{0}\right) \cosh \left(\frac{t}{z_{0}}\right)\right) .
\end{aligned}
$$

For $g_{1}=0$, corresponding $V=0$ in $D$ dimensions, it follows from (2.23) that we must require $k=1$, and hence

$$
\Lambda_{0}=-\frac{1}{4}(D-3)^{2} \varphi_{0}^{-2} .
$$

In this case, the two-dimensional solutions can be lifted to become $D$-dimensional ones. In the Einstein frame, they take the form

$$
\begin{aligned}
d \hat{s}^{2} & =\Phi \frac{2}{D-2}\left(-f d t^{2}+\frac{1}{f} d r^{2}+\varphi_{0}^{2} d \Omega_{D-2}^{2}\right), \\
F & =\sqrt{(D-1)(D-3)} \varphi_{0}^{-1} \Phi d t \wedge d r, \\
& =\phi-\sqrt{\frac{2(D-1)}{D-2}} \log \Phi .
\end{aligned}
$$

For $g_{1} \neq 0$, we can have all $k=1,0,-1$, and $\Lambda_{0}$ is given by (2.23). The $D$-dimensional solutions become

$$
\begin{aligned}
d \hat{s}^{2} & =\Phi \frac{2}{D-2}\left(-f d t^{2}+\frac{1}{f} d r^{2}+\varphi_{0}^{2} d \Omega_{D-2, k}^{2}\right), \\
F & =\sqrt{(D-1)(D-3)\left(k+g_{1}^{2} \varphi_{0}^{2}\right)} \varphi_{0}^{-1} \Phi d t \wedge d r, \\
\phi & =-\sqrt{\frac{2(D-1)}{D-2}} \log \Phi .
\end{aligned}
$$

\subsection{Time-dependent extremal black holes}

The general EMD theory (2.1) admits a class of charged black hole solutions [58]. For the dilaton coupling choice (2.4) and vanishing scalar potential, the charged extremal black hole is given by

$$
\begin{aligned}
d s^{2}= & -H^{-\frac{D-1}{D-2}} \mathcal{H}^{-\frac{D-3}{D-2}} d t^{2} \\
& +H^{\frac{D-1}{(D-2)(D-3)}} \mathcal{H}^{\frac{1}{D-2}}\left(d r^{2}+r^{2} d \Omega_{D-2}^{2}\right), \\
A= & \sqrt{\frac{D-1}{D-3}} H^{-1} d t, \quad \mathcal{A}=\mathcal{H}^{-1} d t, \\
\phi= & \frac{1}{2} \frac{D-1}{D-3} a \log H+\frac{1}{2} b \log \mathcal{H},
\end{aligned}
$$

where $H=1+q / r^{D-3}$ and $\mathcal{H}=1+\tilde{q} / r^{D-3}$ are harmonic functions in the transverse Euclidean space. It was observed in [62] that the harmonic function $\mathcal{H}$ can have a linear timedependence, namely

$\mathcal{H}=h t+\frac{\tilde{q}}{r^{D-3}}$.

Note that here only $\mathcal{H}$, not $H$ can allow such a linear time dependence. After the conformal transformation (2.8), the solution becomes

$$
\begin{aligned}
d s^{2} & =-\frac{d t^{2}}{H \mathcal{H}}+H^{\frac{2}{D-3}}\left(d r^{2}+r^{2} d \Omega_{D-2}^{2}\right), \\
A & =\sqrt{\frac{D-1}{D-3}} H^{-1} d t, \quad \mathcal{A}=\mathcal{H}^{-1} d t, \quad \Phi=\sqrt{\frac{\mathcal{H}}{H}} .
\end{aligned}
$$

To make contact with the solutions in JT gravity, we set $\tilde{q}=0$ and furthermore we take the decoupling limit with 1 in $H$ dropped. The solution becomes

$$
\begin{aligned}
& d s^{2}=-\frac{r^{D-3}}{h r_{0}^{D-3}} d \tilde{t}^{2}+\frac{r_{0}^{2}}{r^{2}} d r^{2}+r_{0}^{2} d \Omega_{D-2}^{2}, \\
& \Phi=\frac{1}{2} \tilde{t} \sqrt{\frac{h r^{D-3}}{r_{0}^{D-3}}}, \quad q=r_{0}^{D-3},
\end{aligned}
$$

where we have redefined the time coordinate by $\tilde{t}=2 \sqrt{t}$. Comparing with the Kaluza-Klein reduction ansatz (3.6), we see that $\varphi_{0}=r_{0}$. Performing the Kaluza-Klein reduction on the $S^{D-2}$ sphere, and redefining the $r$ coordinate and parameters by

$\tilde{r}=\frac{r^{\frac{D-3}{2}}}{\sqrt{\gamma(D-3) r_{0}^{D-4}}}, \quad h=\frac{4 \gamma r_{0}}{D-3}$,

we arrive, after dropping all the tildes, at the two-dimensional solution (3.2) of JT gravity with $\alpha=0$. 


\section{Embeddings in strings and M-theory}

In the previous sections, we show that JT gravity can be obtained from consistent Kaluza-Klein reduction of a class of EMD theory (2.11) in general $D$ dimensions. In four and five dimensions, the EMD theories can be embedded in supergravities, allowing exact embeddings of JT gravity in strings and M-theory. This will provide a better understanding of the time-dependence of the solutions.

\subsection{The $D=5$ theory}

We first consider the $D=5$ EMD theory (2.11). It follows from the discussion in Sect. 2 that in the Einstein frame, the Lagrangian is given by

$$
\mathcal{L}_{5}=\sqrt{-g}\left(R-\frac{1}{2}(\partial \phi)^{2}-\frac{1}{4} e^{\frac{2}{\sqrt{6}} \phi} F^{2}+8 g_{1}^{2} e^{-\frac{1}{\sqrt{6}} \phi}\right),
$$

For $g_{1}=0$, the theory can be embedded into the $U(1)^{3}$ supergravity with two field strengths set equal whilst the third set zero. The theory can be obtained from $\mathcal{N}=(1,0)$ supergravity in $D=6$ via Kaluza-Klein reduction on $S^{1}$ [63]. The relevant six-dimensional theory is Einstein theory coupled to a self-dual 3-form $F_{(3)}=* F_{(3)}$, with

$$
R_{M N}=\frac{1}{4} F_{M P Q} F_{N}^{P Q} .
$$

The (truncated) reduction ansatz is given by

$$
d s_{6}^{2}=e^{\frac{1}{\sqrt{6}} \phi} d s_{5}^{2}+e^{-\frac{3}{\sqrt{6}} \phi} d z^{2}, \quad F_{(3)}=\frac{1}{\sqrt{2}}\left(F \wedge d z+*_{5} F\right) .
$$

The $D=5$ time-dependent extremal black hole (3.7) with $\tilde{q}=0$ becomes

$$
\begin{aligned}
d s_{6}^{2} & =H^{-1}\left(-\mathcal{H}^{-1} d t^{2}+\mathcal{H} d z^{2}\right)+H\left(d r^{2}+r^{2} d \Omega_{3}^{2}\right), \\
F_{(3)} & =-\frac{H^{\prime}}{H^{2}} d t \wedge d z \wedge d r+2 q \Omega_{(3)} \\
H & =1+\frac{q}{r^{2}}, \quad \mathcal{H}=h t
\end{aligned}
$$

This is the self-dual string, with the flat worldsheet metric being the two-dimensional Milne universe, namely

$-\mathcal{H}^{-1} d t^{2}+\mathcal{H} d z^{2}=-h^{-1} d \tilde{t}^{2}+\frac{1}{4} h \tilde{t}^{2} d z^{2}$,

where $t=\frac{1}{4} \tilde{t}^{2}$. The solution can be further lifted to become the intersecting D1/D5 system, or M2/M5-branes. The near horizon geometry has an $\mathrm{AdS}_{3}$ factor whose boundary is the two-dimensional Milne universe rather than the Minkowski spacetime.
We can also lift the full two-dimensional solution (3.2) back to $D=6$ directly, and we find

$d s_{6}^{2}=-\varphi_{0}^{2} r^{2} d t^{2}+\Phi(r, t)^{2} d z^{2}+\frac{d r^{2}}{\varphi_{0}^{2} r^{2}}+\varphi_{0}^{2} d \Omega_{3}^{2}$,

$F_{(3)}=2 \varphi_{0}^{-1} \Phi d t \wedge d r \wedge d z+2 \varphi_{0}^{2} \Omega_{(3)}$.

In particular, when $\alpha=0$, this is precisely the near-horizon geometry of the Milne self-dual string discussed above.

4.2 The $D=4$ theory

The EMD theory (2.11) in four dimensions in the Einstein frame is given by

$\mathcal{L}_{4}=\sqrt{-g}\left(R-\frac{1}{2}(\partial \phi)^{2}-\frac{1}{4} e^{\frac{1}{\sqrt{3}} \phi} F^{2}+3 g_{1}^{2} e^{-\frac{1}{\sqrt{3}} \phi}\right)$,

For $g_{1}=0$, the theory can be embedded into the STU supergravity model, with three equal gauge potentials set equal and the fourth one set zero. The embedding of JT gravity in this theory is part of more general Kaluza-Klein reductions obtained in [53]. The $D=4$ theory $\left(g_{1}=0\right)$ can be obtained from the $S^{1}$ reduction of minimal supergravity in five dimensions, with

$d s_{5}^{2}=e^{-\frac{1}{\sqrt{3}} \phi} d s_{4}^{2}+e^{\frac{2}{\sqrt{3}} \phi} d z^{2}$.

The five-dimensional Maxwell field descends down to four dimensions directly. The time-dependent black hole solution becomes

$$
\begin{aligned}
d s_{5}^{2} & =-H^{-2} d t^{2}+H\left(h t\left(d r^{2}+r^{2} d \Omega_{2}^{2}\right)+\frac{1}{h t} d z^{2}\right), \\
A & =\sqrt{3} H^{-1} d t .
\end{aligned}
$$

Turning off the charge by setting $q=0$, and hence $H=1$, the metric describes a Kasner-type cosmological solution. We can also lift the solution (3.5) to five dimensions and find

$$
\begin{aligned}
d s_{5}^{2} & =\Phi^{2}\left(-\frac{r^{2}}{4 \varphi_{0}^{2}} d t^{2}+\frac{4 \varphi_{0}^{2}}{r^{2}} d r^{2}+\varphi_{0}^{2} d \Omega_{2}^{2}\right)+\Phi^{-2} d z^{2} \\
F & =\sqrt{3} \varphi_{0}^{-1} \Phi(r, t) d t \wedge d r,
\end{aligned}
$$

where $\Phi$ is given in (3.2). This solution with $\alpha=0$ is precisely the decoupling limit of (4.9) with the 1 in $H$ dropped.

The $D=4$ EMD theory with $g_{1}=0$ can also be embedded in M-theory with the reduction ansatz given by

$$
\begin{aligned}
d s_{11}^{2} & =e^{-\frac{1}{\sqrt{3}} \phi} d s_{4}^{2}+e^{\frac{2}{\sqrt{3}} \phi} d z^{2}+d \Sigma_{6}^{2}, \\
A_{(3)} & =\frac{1}{\sqrt{3}} A d t \wedge I_{(2)},
\end{aligned}
$$

where $d \Sigma_{6}^{2}$ is Euclidean or Ricci-flat Calabi-Yau space with a harmonic 2 -form $I_{(2)}$. The oxidized solution can be viewed as 
the $\mathrm{M} 2 / \mathrm{M} 2 / \mathrm{M} 2$ intersection, or M2-brane wrapping around the Calabi-Yau 2-cycles.

We have examined the EMD theories (2.11) for $D=4$ and 5 , with $g_{1}=0$. These theories can be embedded into supergravities. This provides many routes of JT gravity to strings and M-theory, since there are many different ways of embedding of these EMD theories in the fundamental theories (see e.g. [64]). The higher dimensional solutions are related to M-branes, D-branes and their intersections, see e.g. [65-67]

\section{$4.3 D=4,5$ theories with $g_{1} \neq 0$}

When $g_{1} \neq 0$, the theory can also be obtained from taking an appropriate limit of gauged supergravities, by taking $g_{2}=0$ in the scalar potential (2.7). However, the sphere reduction ansätze constructed for $g_{1} g_{2} \neq 0$ [59] do not appear to allow the $g_{2}=0$ limit. It turns out that although the $D=4,5$ theories with $g_{2}=0$ and $g_{1} \neq 0$ cannot be obtained Mtheory or strings directly from sphere reductions, they can be obtained from $S^{1}$ reduction of gauged supergravities that can be reduced from higher dimensions on spheres.

For example, the Lagrangian for the bosonic sector of minimal gauged supergravity in five dimensions is

$$
\mathcal{L}_{5}=\sqrt{-g}\left(R-\frac{1}{4} F^{2}+12 g^{2}\right)+\frac{1}{12 \sqrt{3}} \varepsilon^{\mu \nu \rho \sigma \lambda} F_{\mu \nu} F_{\rho \sigma} A_{\lambda} .
$$

For the pure electric ansatz we consider in this paper, the last $F F A$ term can be dropped. The reduction ansatz (4.8) with the $F$ descending directly gives rise to precisely the $D=4$ EMD theory (4.7), with $g_{1}=2 g$. The lifting of the (3.2) leads to

$$
\begin{aligned}
d s_{5}^{2}= & -\frac{1}{4}\left(k \varphi_{0}^{2}+3 g_{1}^{2}\right) r^{2} d t^{2} \\
& +\frac{4}{\left(k \varphi_{0}^{2}+3 g_{1}^{2}\right) r^{2}} d r^{2}+\varphi_{0}^{2} d \Omega_{2, k}^{2}+\Phi^{2} d z^{2} \\
F= & \sqrt{3\left(k+g_{1}^{2} \varphi_{0}^{2}\right)} \varphi_{0} \Omega_{(2), k}
\end{aligned}
$$

The five-dimensional gauged supergravity (4.12) can itself be obtained from type IIB supergravity on $S^{5}$, and the reduction ansatz can be found in [59]. Its (singular) embedding in M-theory was also obtained [68]. The effective cosmological constant $\Lambda_{0}$ (2.23) in JT gravity now has contributions from D3-brane charges as well as the gauged supergravity R-charges associated with rotations of D3-branes.

The $D=5$ theory can also be obtained from $D=6$ Einstein gravity coupled to cosmological constant and a selfdual 3-form

$$
R_{M N}=\frac{1}{4} F_{M P Q} F_{N}^{P Q}-5 g^{2} g_{M N}, \quad F_{(3)}=* F_{(3)} .
$$

The reduction ansatz (4.3) gives precisely (4.1) with $g_{1}^{2}=$ $5 g^{2} / 2$. The resulting solution is given by

$$
\begin{aligned}
d s_{6}^{2}= & -\left(k \varphi_{0}^{-2}+2 g_{1}^{2}\right) r^{2} d t^{2}+\frac{1}{\left(k \varphi_{0}^{-2}+2 g_{1}^{2}\right) r^{2}} d r^{2} \\
& +\varphi_{0}^{2} d \Omega_{3, k}^{2}+\Phi^{2} d z^{2} \\
F= & 2 \sqrt{k+g_{1}^{2} \varphi_{0}^{2}}\left(\varphi_{0}^{-1} \Phi d t \wedge d r \wedge d z+\varphi_{0}^{2} \Omega_{(3), k}\right)
\end{aligned}
$$

The (4.14) theory is a consistent truncation of the bosonic sector of $D=6, \mathcal{N}=(1,1)$ gauged supergravity that can be embedded in massive type IIA theory [69]. The effective cosmological constant in JT gravity in this embedding is related to the D4/D8-brane charges.

Finally, it is worth noting that for $F=0$, the theory (2.11) in general $D$ dimensions can all be obtained from the circle reduction of Einstein gravity with a cosmological constant in $D+1$ dimensions [60].

\section{Conclusions}

In this paper, we demonstrated that JT gravity in two dimensions could be obtained from the consistent Kaluza-Klein reduction on a class of EMD theories (2.11) in general $D$ dimensions. For $D=4$ and 5, the EMD theories are truncations of the bosonic sector of supergravities. This allows one to embed JT gravities in strings or M-theory, providing stringy interpretations of the SYK model.

The exact embeddings of this paper also allow one to understand the solutions of JT gravity in the light of higher dimensional theories. For $g_{1}=0$, we find that a class of JT gravity solutions are related to the time-dependent extremal charged black holes in the EMD theories. The solutions can be further lifted to become, for example, intersecting D1/D5brane, where the worldsheet is the $2 \mathrm{~d}$-Milne universe instead of the more traditional 2d-Minkowski spacetime. They can also be lifted to the time-dependent M2/M2/M2 intersections. For $g_{1} \neq 0$, we find that the cosmological constant in JT gravity is related to D3-brane or D4/D8-brane charges depending on the specific route of the embeddings.

The exact embeddings of this paper imply that we can obtain the Schwarzian action directly in higher dimensions. The subtlety is that the worldsheet or worldvolume of branes should be described by the Milner metric rather than the usual Minkowski metric. The fact that the Milne universe appears in the worldsheet or worldvolume is tantalizing. Nondilatonic extremal $p$-branes such as the M-branes or the D3brane have in general $\mathrm{AdS}_{d} \times S^{D-d}$ as their near-horizon geometry. When the boundary (the brane worldvolume) of the $\operatorname{AdS}_{d}$ is the Milne universe, we have 


$$
\begin{aligned}
d s_{d}^{2} & =\frac{d r^{2}}{r^{2}}+r^{2}\left(-d t^{2}+t^{2} d \Omega_{d-2,-1}^{2}\right) \\
& =\left(-r^{2} d t^{2}+\frac{d r^{2}}{r^{2}}\right)+\Phi^{2} d \Omega_{d-2,-1}^{2},
\end{aligned}
$$

where $d \Omega_{d-2,-1}^{2}$ is taken to be some compact metrics with negative cosmological constant. The Kaluza-Klein reduction on $d \Omega_{d-2,-1}^{2}$ yields naturally a two-dimensional gravity with nearly $\mathrm{AdS}_{2}$ vacuum geometry. It would be of great interest to investigate the corresponding holographic $\mathrm{NCFT}_{1}$.

Acknowledgements Y.Z.L. and H.L. are supported in part by NSFC grants Nos. 11475024 and 11235003; S.L.L. is supported in part by NSFC under Grants Nos. 11575022 and 11175016 . We are grateful to Eoin Colgáin, Yong-Hui Qi, Zhao-Long Wang and Hossein Yavartanoo for useful discussions.

Open Access This article is distributed under the terms of the Creative Commons Attribution 4.0 International License (http://creativecomm ons.org/licenses/by/4.0/), which permits unrestricted use, distribution, and reproduction in any medium, provided you give appropriate credit to the original author(s) and the source, provide a link to the Creative Commons license, and indicate if changes were made.

Funded by SCOAP ${ }^{3}$.

\section{A From JT gravity to SYK}

In this appendix, we give a detail review of how the SYK model can arise from JT gravity. This review is based on the work of [34]. JT gravity admits the nearly $\mathrm{AdS}_{2}$ solution (3.2). In the Euclidean signature, the solution reads

$d s^{2}=\frac{d t^{2}+d z^{2}}{z^{2}}, \quad \Phi=\alpha z+\frac{\beta+\gamma t+\Lambda_{0}^{2} \alpha t^{2}}{z}$

where $z=1 / r$ and we have chosen $\Lambda_{0}=-1$ without loss of generality.

For the theory to be well-defined, we need to include the Gibbons-Hawking surface term and the appropriate counterterm to the action (1.2). The total action is given by (after setting $\left.\Lambda_{0}=-1\right)$ :

$S=\frac{1}{16 \pi} \int_{M} d^{2} x \sqrt{g} \Phi(R+2)+\frac{1}{8 \pi} \int_{\partial M} \sqrt{h} \Phi(K-1)$,

where $h$ is the induced metric on the relevant boundary. Note that we also included the holographic counterterm above, as was done in [70].

Following [34,37], we slice the original asymptotic boundary in terms of a new parameter $u$. In other words, the new boundary deviates from the AdS fixed point and is parameterized by $(t(u), z(u))$. The variables $(t(u), z(u))$ are then interpreted as the dynamical fields of the boundary, where $u$ naturally serves as the Euclidean time of the boundary CFT. It is clear that the new boundary is fixed by $g_{u u}=\frac{1}{\epsilon^{2}}=\frac{t^{\prime 2}+z^{\prime 2}}{z^{2}}$

where a prime denotes a derivative with respect to $u$, and $\epsilon$ is an infinitesimal constant representing the UV cutoff. Equation (A.3) implies that $t(u)$ and $z(u)$ are not independent dynamic fields asymptotically, they are related by

$z(u)=\epsilon t^{\prime}+\frac{1}{2} \epsilon^{3} \frac{t^{\prime \prime 2}}{t^{\prime}}+\cdots, \quad \Phi \rightarrow \frac{\Phi_{\text {inf }}}{\epsilon}$,

where $\Phi_{\text {inf }}$ is given by

$\Phi_{\text {inf }}=\frac{\beta+\gamma t+\alpha t^{2}}{t^{\prime}}$

The rigid AdS geometry causes the bulk action to vanish identically, and the total action will be given by the boundary action, namely

$S=\frac{1}{8 \pi} \int d u \frac{\Phi_{\text {inf }}}{\epsilon^{2}}(K-1), \quad K=h^{\mu v} \nabla_{\mu} n_{v}$.

To evaluate the vector $n=n^{\mu} \partial_{\mu}$ normal to the boundary, we note that the tangent vector is given by

$$
\begin{aligned}
T & =\frac{\partial}{\partial u}=t^{\prime} \frac{\partial}{\partial t}+\epsilon t^{\prime \prime}\left(1+\epsilon^{2}\left(\frac{t^{\prime \prime \prime}}{t^{\prime}}-\frac{1}{2}\left(\frac{t^{\prime \prime}}{t^{\prime}}\right)^{2}\right)\right) \frac{\partial}{\partial z} \\
& \equiv T^{\mu} \partial_{\mu},
\end{aligned}
$$

where we have used (A.4). Since the normal vector is defined by

$T^{\mu} n_{\mu}=0, \quad n_{\mu} n_{v} g^{\mu \nu}=1$,

We find, up to the $\epsilon^{2}$ order, that

$n_{0}=\frac{t^{\prime \prime}}{t^{\prime 2}}\left(1+\epsilon^{2} \operatorname{Sch}(t(u), u)\right), \quad n_{1}=-\frac{1}{\epsilon t}\left(1-\epsilon^{2}\left(\frac{t^{\prime \prime}}{t^{\prime}}\right)^{2}\right)$,

where $\operatorname{Sch}(t(u), u)$ is the Schwarzian derivative

$\operatorname{Sch}(t(u), u)=\frac{2 t^{(3)} t^{\prime}-3 t^{\prime \prime 2}}{2 t^{\prime 2}}$

The normal vector $n^{\mu}$, the tangent vector $T^{\mu}$ and the metric $g_{\mu \nu}$ satisfy the identity

$g_{\mu \nu}-n_{\mu} n_{v}=\frac{T_{\mu} T_{v}}{T^{2}}$

It follows that the extrinsic curvature can be rewritten as

$K=\frac{T^{\mu}}{T^{2}}\left(n_{\mu}^{\prime}-n_{\rho} \Gamma_{\mu \nu}^{\rho} T^{v}\right)$ 
Substituting (A.7) and (A.9) into (A.12), we find

$$
K=1+\operatorname{Sch}(t(u), u) \epsilon^{2}+\cdots,
$$

where the dots represent those with higher powers of $\epsilon$. Therefore, it is straightforward to see that (A.6) is given by

$S=\frac{1}{8 \pi} \int d u \Phi_{\text {inf }} \operatorname{Sch}(t(u), u)$

From (A.17), varying $t(u)$, we can obtain solution for $\Phi_{\text {inf }}(u)$, i.e. (A.5). It should be emphasized that $\Phi_{\text {inf }}$ here should be viewed as a background rather than a dynamical field; it does not involve in the variation principle. The Eq. (A.5) is thus an equation of motion for $t(u)$ at a given background $\Phi_{\text {inf }}(u)$. Making a coordinate transformation

$\Phi_{\inf } \frac{\partial \tilde{u}}{\partial u}=\Phi_{c}$,

where $\Phi_{c}$ is a constant, we find

$S=\frac{\Phi_{c}}{8 \pi} \int d \tilde{u}(\operatorname{Sch}(t(\tilde{u}), \tilde{u})-\operatorname{Sch}(u(\tilde{u}), \tilde{u}))$.

The second term in the bracket is a quantity that has no dynamical field and can be dropped, leading to the Schwarzian action

$S=\frac{\Phi_{c}}{8 \pi} \int d u \operatorname{Sch}(t(u), u)$.

It is the effective action describing the IR behavior of the SYK model [5], and is invariant under the $S L(2, \mathbb{R})$ transformation

$t(u) \rightarrow \frac{a t(u)+b}{c t(u)+d}, \quad a d-b c=1$.

It is of interest to note that had one considered $\Phi_{\text {inf }}$ as a dynamical field, then Eq. (A.16) would imply that the system had a ghost excitation.

\section{References}

1. J.M. Maldacena, The large $N$ limit of superconformal field theories and supergravity. Int. J. Theor. Phys. 38, 1113 (1999)

2. J.M. Maldacena, Adv. Theor. Math. Phys. 2, 231 (1998). https:// doi.org/10.1023/A:1026654312961. arXiv:hep-th/9711200

3. S. Sachdev, J. Ye, Gapless spin fluid ground state in a random, quantum Heisenberg magnet. Phys. Rev. Lett. 70, 3339 (1993). https:// doi.org/10.1103/PhysRevLett.70.3339. arXiv:cond-mat/9212030

4. A. Kitaev, A simple model of quantum holography, KITP strings seminar and Entanglement 2015 program (Feb. 12, April 7, and May 27, 2015). http://online.kitp.ucsb.edu/online/entangled15/. Accessed 1 Oct 2017
5. J. Maldacena, D. Stanford, Remarks on the Sachdev-Ye-Kitaev model. Phys. Rev. D 94(10), 106002 (2016). https://doi.org/10. 1103/PhysRevD.94.106002. arXiv:1604.07818 [hep-th]

6. J. Polchinski, V. Rosenhaus, The spectrum in the Sachdev-YeKitaev model. JHEP 1604, 001 (2016). https://doi.org/10.1007/ JHEP04(2016)001. arXiv:1601.06768 [hep-th]

7. S.H. Shenker, D. Stanford, Black holes and the butterfly effect. JHEP 1403, 067 (2014). https://doi.org/10.1007/ JHEP03(2014)067. arXiv:1306.0622 [hep-th]

8. J. Maldacena, S.H. Shenker, D. Stanford, A bound on chaos. JHEP 1608, 106 (2016). https://doi.org/10.1007/JHEP08(2016)106. arXiv:1503.01409 [hep-th]

9. A.M. Garca-Garca, J.J.M. Verbaarschot, Spectral and thermodynamic properties of the Sachdev-Ye-Kitaev model. Phys. Rev. D 94(12), 126010 (2016). https://doi.org/10.1103/PhysRevD.94. 126010. arXiv:1610.03816 [hep-th]

10. R.A. Davison, W. Fu, A. Georges, Y. Gu, K. Jensen, S. Sachdev, Thermoelectric transport in disordered metals without quasiparticles: the Sachdev-Ye-Kitaev models and holography. Phys. Rev. B 95(15), 155131 (2017). https://doi.org/10.1103/PhysRevB.95. 155131. arXiv:1612.00849 [cond-mat.str-el]

11. S.K. Jian, H. Yao, Solvable Sachdev-Ye-Kitaev models in higher dimensions: from diffusion to many-body localization. Phys. Rev. Lett 119(20), 206602 (2017). https://doi.org/10.1103/ PhysRevLett.119.206602. arXiv:1703.02051 [cond-mat.str-el]

12. D. Stanford, E. Witten, Fermionic localization of the Schwarzian theory. JHEP 1710, 008 (2017). https://doi.org/10.1007/ JHEP10(2017)008. arXiv:1703.04612 [hep-th]

13. T.G. Mertens, G.J. Turiaci, H.L. Verlinde, Solving the Schwarzian via the conformal bootstrap. JHEP 1708, 136 (2017). https://doi. org/10.1007/JHEP08(2017)136. arXiv:1705.08408 [hep-th]

14. M. Taylor, Generalized conformal structure, dilaton gravity and SYK. JHEP 1801, 010 (2018). https://doi.org/10.1007/ JHEP01(2018)010. arXiv:1706.07812 [hep-th]

15. I. Kourkoulou, J. Maldacena, Pure states in the SYK model and nearly-AdS 2 gravityarXiv:1707.02325 [hep-th]

16. R. Bhattacharya, S. Chakrabarti, D.P. Jatkar, A. Kundu, SYK model, chaos and conserved charge. JHEP 1711, 180 (2017). https://doi.org/10.1007/JHEP11(2017)180. arXiv:1709.07613 [hep-th]

17. A. Kitaev, S.J. Suh, The soft mode in the Sachdev-Ye-Kitaev model and its gravity dual. arXiv:1711.08467 [hep-th]

18. A.M. Garca-Garca, Y. Jia, J.J.M. Verbaarschot, Exact moments of the Sachdev-Ye-Kitaev model up to order 1/ $N^{2}$. arXiv:1801.02696 [hep-th]

19. A. M. Garca-Garca, M. Tezuka, Many-body localization in a finiterange Sachdev-Ye-Kitaev model. arXiv:1801.03204 [hep-th]

20. I. Aref 'eva, I. Volovich, Notes on the SYK model in real time. arXiv: 1801.08118 [hep-th]

21. D.A. Roberts, D. Stanford, A. Streicher, Operator growth in the SYK model. arXiv:1802.02633 [hep-th]

22. Y.H. Qi, Y. Seo, S.J. Sin, G. Song, Schwarzian correction to quantum correlation in SYK model. arXiv:1804.06164 [hep-th]

23. W. Fu, D. Gaiotto, J. Maldacena, S. Sachdev, Supersymmetric Sachdev-Ye-Kitaev models, Phys. Rev. D 95 no.2, 026009 (2017) Addendum: [Phys. Rev. D 95 (2017) no.6, 069904] https://doi.org/10.1103/PhysRevD.95.069904, https://doi.org/10. 1103/PhysRevD.95.026009 [arXiv:1610.08917 [hep-th]]

24. T. Li, J. Liu, Y. Xin, Y. Zhou, Supersymmetric SYK model and random matrix theory. JHEP 1706, 111 (2017). https://doi.org/10. 1007/JHEP06(2017)111. arXiv:1702.01738 [hep-th]

25. J. Murugan, D. Stanford, E. Witten, More on supersymmetric and 2d analogs of the SYK model. JHEP 1708, 146 (2017). https://doi. org/10.1007/JHEP08(2017)146. arXiv:1706.05362 [hep-th] 
26. N. Hunter-Jones, J. Liu, Y. Zhou, On thermalization in the SYK and supersymmetric SYK models. JHEP 1802, 142 (2018). https:// doi.org/10.1007/JHEP02(2018)142. arXiv:1710.03012 [hep-th]

27. K. Bulycheva, " $\mathcal{N}=2$ SYK model in the superspace formalism," arXiv:1801.09006 [hep-th]

28. Y. Gu, X.L. Qi, D. Stanford, Local criticality, diffusion and chaos in generalized Sachdev-Ye-Kitaev models. JHEP 1705, 125 (2017). https://doi.org/10.1007/JHEP05(2017)125. arXiv: 1609.07832 [hep-th]

29. D.J. Gross, V. Rosenhaus, A Generalization of SachdevYe-Kitaev. JHEP 1702, 093 (2017). https://doi.org/10.1007/ JHEP02(2017)093. arXiv:1610.01569 [hep-th]

30. W. Cai, X.H. Ge, G.H. Yang, Diffusion in higher dimensional SYK model with complex fermions. JHEP 1801, 076 (2018). https://doi. org/10.1007/JHEP01(2018)076. arXiv:1711.07903 [hep-th]

31. C. Teitelboim, Gravitation and Hamiltonian structure in two spacetime dimensions. Phys. Lett. 126B, 41 (1983). https://doi.org/10. 1016/0370-2693(83)90012-6

32. R. Jackiw, Lower dimensional gravity. Nucl. Phys. B 252, 343 (1985). https://doi.org/10.1016/0550-3213(85)90448-1

33. J. Engelsoy, T.G. Mertens, H. Verlinde, An investigation of $\mathrm{AdS}_{2}$ backreaction and holography. JHEP 1607, 139 (2016). https://doi. org/10.1007/JHEP07(2016)139. arXiv:1606.03438 [hep-th]

34. J. Maldacena, D. Stanford, Z. Yang, Conformal symmetry and its breaking in two dimensional Nearly Anti-de-Sitter space. PTEP 2016(12), 12C104 (2016). https://doi.org/10.1093/ptep/ptw124. arXiv:1606.01857 [hep-th]

35. M. Cadoni, M. Ciulu, M. Tuveri, Symmetries, holography and quantum phase transition in two-dimensional dilaton AdS gravityarXiv:1711.02459 [hep-th]

36. K. Jensen, Chaos in $\mathrm{AdS}_{2}$ holography. Phys. Rev. Lett. 117(11), 111601 (2016). https://doi.org/10.1103/PhysRevLett.117.111601. arXiv:1605.06098 [hep-th]

37. S. Forste, I. Golla, Nearly $\mathrm{AdS}_{2}$ sugra and the super-Schwarzian. Phys. Lett. B 771, 157 (2017). https://doi.org/10.1016/j.physletb. 2017.05.039. arXiv:1703.10969 [hep-th]

38. S. Forste, J. Kames-King, M. Wiesner, "Towards the holographic dual of $\mathcal{N}=2$ SYK," https://doi.org/10.1007/JHEP03(2018)028 arXiv: 1712.07398 [hep-th]

39. H.A. González, D. Grumiller, J. Salzer, Towards a bulk description of higher spin SYK. arXiv:1802.01562 [hep-th]

40. J. Maldacena, X.L. Qi, Eternal traversable wormhole, arXiv: 1804.00491 [hep-th]

41. D. Grumiller, W. Kummer, D.V. Vassilevich, Dilaton gravity in two-dimensions. Phys. Rept. 369, 327 (2002). https://doi.org/10. 1016/S0370-1573(02)00267-3. arXiv:hep-th/0204253

42. A. Almheiri, J. Polchinski, Models of $\mathrm{AdS}_{2}$ backreaction and holography. JHEP 1511, 014 (2015). https://doi.org/10.1007/ JHEP11(2015)014. arXiv:1402.6334 [hep-th]

43. G. Sárosi, $\mathrm{AdS}_{2}$ holography and the SYK model. PoS Modave 2017, 001 (2018). arXiv:1711.08482 [hep-th]

44. C.G. Callan Jr., S.B. Giddings, J.A. Harvey, A. Strominger, Evanescent black holes. Phys. Rev. D 45(4), R1005 (1992). https://doi.org/ 10.1103/PhysRevD.45.R1005. arXiv:hep-th/9111056

45. S.B. Giddings, A. Strominger, Dynamics of extremal black holes. Phys. Rev. D 46, 627 (1992). https://doi.org/10.1103/PhysRevD. 46.627. arXiv:hep-th/9202004

46. M. Cadoni, S. Mignemi, Classical and semiclassical properties of extremal black holes with dilaton and modulus fields. Nucl. Phys. B 427, 669 (1994). https://doi.org/10.1016/0550-3213(94)90644-0. arXiv:hep-th/9312171

47. M. Cadoni, S. Mignemi, Nonsingular four-dimensional black holes and the Jackiw-Teitelboim theory. Phys. Rev. D 51, $4319 \quad$ (1995). https://doi.org/10.1103/PhysRevD.51.4319. arXiv:hep-th/9410041
48. K.S. Kolekar, K. Narayan, " $\mathrm{AdS}_{2}$ dilaton gravity from reductions of some nonrelativistic theories," arXiv:1803.06827 [hep-th]

49. J. Michelson, Anti-de Sitter fragmentation. arXiv:hep-th/9909069

50. A. Almheiri, Magnetic $\mathrm{AdS}_{2} \times R_{2}$ at weak and strong coupling. arXiv: 1112.4820 [hep-th]

51. P. Nayak, A. Shukla, R.M. Soni, S.P. Trivedi and V. Vishal, On the dynamics of near-extremal black holes. arXiv: 1802.09547 [hep-th]

52. A. Almheiri, B. Kang, Conformal symmetry breaking and thermodynamics of near-extremal black holes. JHEP 1610, 052 (2016). https://doi.org/10.1007/JHEP10(2016)052. arXiv:1606.04108 [hep-th]

53. M. Cvetič, I. Papadimitriou, "AdS 2 holographic dictionary," JHEP 1612008 (2016) Erratum: [JHEP 1701 (2017) 120] https:// doi.org/10.1007/JHEP12(2016)008, https://doi.org/10.1007/ JHEP01(2017)120 [arXiv:1608.07018 [hep-th]]

54. S.R. Das, A. Jevicki, K. Suzuki, Three dimensional view of the SYK/AdS duality. JHEP 1709, 017 (2017). https://doi.org/10. 1007/JHEP09(2017)017. arXiv:1704.07208 [hep-th]

55. T.G. Mertens, "The Schwarzian theory - origins," arXiv:1801.09605 [hep-th]

56. A. Gaikwad, L.K. Joshi, G. Mandal, S.R. Wadia, "Holographic dual to charged SYK from 3D gravity and Chern-Simons," arXiv:1802.07746 [hep-th]

57. M.J. Duff, J.T. Liu, J. Rahmfeld, Four-dimensional string-stringstring triality. Nucl. Phys. B 459, 125 (1996). https://doi.org/10. 1016/0550-3213(95)00555-2. arXiv:hep-th/9508094

58. H. Lü, Charged dilatonic ads black holes and magnetic $\operatorname{AdS}_{D-2} \times$ $R^{2}$ vacua. JHEP 1309, 112 (2013). https://doi.org/10.1007/ JHEP09(2013)112. arXiv:1306.2386 [hep-th]

59. M. Cvetič, M.J. Duff, P. Hoxha, James T. Liu, H. Lü, J.X. Lu, R. Martinez-Acosta, C.N. Pope, H. Sati, Tuan A. Tran, Embedding AdS black holes in ten-dimensions and elevendimensions. Nucl. Phys. B 558, 96 (1999). https://doi.org/10.1016/ S0550-3213(99)00419-8. arXiv:hep-th/9903214

60. H. Liu, H. Lü, Z.L. Wang, $f(R)$ theories of supergravities and pseudo supergravities. JHEP 1204, 072 (2012). https://doi.org/10. 1007/JHEP04(2012)072. arXiv:1201.2417 [hep-th]

61. M.S. Bremer, M.J. Duff, H. Lü, C.N. Pope, K.S. Stelle, Instanton cosmology and domain walls from M-theory and string theory. Nucl. Phys. B 543, 321 (1999). https://doi.org/10.1016/ S0550-3213(98)00764-0. arXiv:hep-th/9807051

62. G.W. Gibbons, H. Lü, C.N. Pope, Brane worlds in collision. Phys. Rev. Lett. 94, 131602 (2005). https://doi.org/10.1103/ PhysRevLett.94.131602. arXiv:hep-th/0501117

63. H. Lü, C.N. Pope, E. Sezgin, K.S. Stelle, Stainless super pbranes. Nucl. Phys. B 456, 669 (1995). https://doi.org/10.1016/ 0550-3213(95)00524-4. arXiv:hep-th/9508042

64. H. Lü, C.N. Pope, $p$-brane solitons in maximal supergravities. Nucl. Phys. B 465, 127 (1996). https://doi.org/10.1016/ 0550-3213(96)00048-X. arXiv:hep-th/9512012

65. A.A. Tseytlin, Harmonic superpositions of M-branes. Nucl. Phys. B 475, 149 (1996). https://doi.org/10.1016/ 0550-3213(96)00328-8. arXiv:hep-th/9604035

66. K. Behrndt, E. Bergshoeff, B. Janssen, Intersecting dbranes in ten-dimensions and six-dimensions. Phys. Rev. D 55, 3785 (1997). https://doi.org/10.1103/PhysRevD.55.3785. arXiv:hep-th/9604168

67. H. Lü, C.N. Pope, T.A. Tran, K.W. Xu, Classification of p-branes, NUTs, waves and intersections. Nucl. Phys. B 511, 98 (1998). https://doi.org/10.1016/S0550-3213(97)00735-9. arXiv:hep-th/9708055

68. E. ó. Colgáin, M.M. Sheikh-Jabbari, J.F. Vázquez-Poritz, H. Yavartanoo, Z. Zhang, Warped Ricci-flat reductions. Phys. Rev. D 90(4), 045013 (2014). https://doi.org/10.1103/PhysRevD.90. 045013. arXiv:1406.6354 [hep-th] 
69. M. Cvetič, H. Lü, C.N. Pope, Gauged six-dimensional supergravity from massive type IIA. Phys. Rev. Lett. 83, 5226 (1999). https:// doi.org/10.1103/PhysRevLett.83.5226. arXiv:hep-th/9906221

70. D. Harlow, D. Jafferis, The factorization problem in JackiwTeitelboim gravity. arXiv:1804.01081 [hep-th] 